Saudi Journal of Biomedical Research

Abbreviated Key Title: Saudi J Biomed Res ISSN 2518-3214 (Print) |ISSN 2518-3222 (Online)

\title{
Physiological Effect of Quercetin as a Natural Flavonoid to be used as Hypoglycemic Agent in Diabetes Mellitus Type II Rats
}

\author{
Osama A. Shaikhomar ${ }^{1 *}$, Omar S. Bahattab ${ }^{2}$ \\ ${ }^{1}$ Department of physiology, Faculty of Medicine, Umm Al-Qura University, Makkah, Saudi Arabia \\ ${ }^{2}$ Biology Department, Faculty of Science, Tabuk University, Tabuk, Saudi Arabia
}

\begin{abstract}
DOI: $10.36348 /$ sjbr.2021.v06i01.003
| Received: 12.01.2021 | Accepted: 25.01.2021 | Published: 30.01.2021
\end{abstract}

*Corresponding author: Osama A. Shaikhomar

\section{Abstract}

Diabetes Mellitus (DM), especially type II; represents a major health problem in Saudi Arabia, due to the dramatic changes in the lifestyles of Saudi population. As per the report of World Health Organization (WHO) for the occurrence of diabetes, Saudi Arabia ranked the second in the Middle East and seventh in the world. The increasing trends of diabetes in Saudi Arabia are more worrying in the last few years. Type II DM is generally characterized by peripheral insulin resistance and relative insulin deficiency. Quercetin is a natural flavonoid which belongs to a group of natural substances with variable phenolic structures, found naturally in vegetables, fruits and especially in high amounts in onions, apples, tea and grape juice. Self-emulsifying drug delivery system (SEDDS) were prepared following the component ratios. Then, the Quercetin was dissolved and the components were mixed and heated at $37^{\circ} \mathrm{C}$ until the drug perfectly has dissolved. The mixture prepared stored at room temperature. The aim of this study was to optimize concentration of Quercetin leading to efficient decrease in blood glucose level in streptozotocin induced diabetes among the treated rats as compared to the reference drug e.g. glibenclamide. The results showed that the blood glucose lowering activity of flavonoid compounds may be due to stimulating $\beta$-cells to release more insulin. The comparative analysis of natural flavonoids showed that the Quercetin was more effective anti-diabetic and hypoglycemic compound.

Keywords: Diabetes Mellitus Type II, Hypoglycemic Agent, Natural Flavonoid, Physiological Effect, Quercetin.

Copyright () 2021 The Author(s): This is an open-access article distributed under the terms of the Creative Commons Attribution 4.0 International License (CC BY-NC 4.0) which permits unrestricted use, distribution, and reproduction in any medium for non-commercial use provided the original author and source are credited.

\section{INTRODUCTION}

Diabetes Mellitus is one of the common metabolic disorders with micro- and macro-vascular complications that results in significant mortality and morbidity [1]. Chronic Diabetes Mellitus is a serious complication that affected approximately 135 million people in 1995, 285 million people in 2010, rising to 463 million people worldwide in 2019 and by 2030 is projected to be 600 million [2]. Diabetes mellitus is among the five leading causes of world's death count [3]. Even in modern system of medicine, there are no satisfactory and effective therapies are available to cure it [4]. Now the patients are more interested in natural anti-diabetic products rather than using insulin and oral hypoglycemic agents due to their side effects [5-7]. There is continuous generation of oxygen reactive species (ROS) in hyperglycemic condition. Over production of oxygen free radicals by ROS increase the oxidative stress that results in development of diabetes. Antioxidants are important in scavenging the free radical, damaging the ROS and thus protects the human body from oxidative stress [8]. Hence, a drug which is antioxidant and anti-diabetic would be very useful for the treatment [9].

Diabetes mellitus is characterized by hyperglycemia and hyperlipidemia. Diabetic patients experience various vascular complications, such as Atherosclerosis, Diabetic Nephropathy, and Neuropathy [10]. Type-II Diabetes Mellitus (NIDDM) is defined as a combination of insulin resistance (reduced ability of insulin to stimulate utilization of glucose in the body), and reduced secretion of insulin [11]. Insulin and use of various other oral anti-diabetic agents are the currently available therapies for diabetes but these drugs are associated with several serious side effects [12, 13]. Hence anti-diabetic drug discovery has shifted its focus to natural plant sources having minimal side effects [10]. To cure diabetes, some medicinal plants with rich source of various chemical constituents acts on a variety of mechanism [2]. 
The epidemiologic shift in the Kingdom of Saudi Arabia (KSA) has been rapid and complex. Fast economic growth in the last four decades led to an exceptional increase in living standards. Adoption of a "Westernized Lifestyle" resulted in unhealthy dietary habits and decrease in physical activity. An increase in the prevalence of NIDDM is observed during this period, which is attributed to the dramatic change in lifestyle. In addition to genetic predisposition of diabetes in Saudi peoples, prevalent consanguineous marriages are also observed.

The most common and preferred method of drug administration is the oral route as it is convenient and ease of ingestion. However, it is a challenge to pharmaceutical scientists for the oral delivery of lipophilic drugs due to their inherent low aqueous solubility resulting into poor oral bioavailability, high intra and inter-subject variability and lack of drug dose proportionality [14]. The pharmaceutical scientists used various methods to improve the dissolution rate of drugs those are poorly water- soluble, including formulations containing nano-particles, a solid solution formulation, cyclodextrin inclusion complex formation and self-emulsifying drug delivery system (SEDDS) $[15,19]$.

SEDDS is an isotropic oil mixture with surfactant and/or co- surfactant diluted in aqueous media, such as gastrointestinal fluids, impulsive formation of emulsions upon mild agitation [20, 21]. In the case of sparingly soluble drugs, the SEDDS system offers a way to improve the rate and extent of oral absorption and produce more reproducible blood-time profiles $[15,22]$. It provides with a large interfacial area for partitioning of drug between oil and water, and also a good interface for lipolytic enzymes to digest the oil to promote rapid drug release between various phases of intestinal contents [23]. SEDDS showed improved physical stability upon long-term storage in absence of water content, so that they can be filled directly into hard or soft gelatin capsules for convenient oral delivery [24].

Flavonoids are from the group of natural substances with varying phenolic structures. They are found in roots, stems, bark, flowers, fruits, vegetables and grains, and. Quercetin is a major member of flavonoid family isolated from onions, apples, grapes and tea. This flavanol is mostly well-known for its antioxidant and anti-inflammatory properties. It has been indicated that Quercetin can be beneficial for preventing hyperglycemia in experimental version of Diabetes Mellitus [25]. Oral administration of $100 \mathrm{mg} / \mathrm{kg}$ Quercetin could weaken fasting and postprandial levels of glucose in experimental Diabetes Mellitus, at least in part by inhibiting $\alpha$-glucosidase activity [26]. In addition, extract of onion skin containing $6.04 \mathrm{~g}$ quercetin/100 g dried weight, significantly reduced the blood glucose spike after sucrose loading [27].

The low aqueous solubility of Quercetin hampers its use as a therapeutic agent ${ }^{(28)}$. Quercetin solubility was determined to be $0.441 \pm 0.0487 \mu \mathrm{g} / \mathrm{mL}$ at room temperature ${ }^{(29)}$. The pharmacokinetics of Quercetin excludes more than $1 \%$ absorption of unchanged drug. Therefore, any attempt to increase the dissolution rate would improve its bio-absorption and bioavailability [30]. The absorption of Quercetin from the gastrointestinal tract (GIT) may be limited to dissolution-rate. Therefore, improvement of Quercetin dissolution characteristics may result in an increase of its bioavailability [31]. Different methods and techniques had been utilized to improve the solubility of Quercetin, these techniques include loading of Quercetin into polymeric micelles [28], nanocrystals [32], microspheres [33], and inclusion complexes with cyclodextrins [34], water soluble polymers as PEG and PVP [31].

Optimal formulation is likely to be obtained, based on pre-formulation studies; solubility, phase diagram, in vitro efficiency of self-emulsificationand in vitro release. This study aims to optimize concentration of Quercetin leading to efficient decrease in blood glucose level in streptozotocin which induce diabetes among the treated rats as compared to the reference drug e.g. glibenclamide.

\section{METHODS}

\section{Phase I: Pre-formulation studies Solubility Studies}

For preparation of successful SEDDS, the drug solubility in different oils, surfactants and cosurfactants were investigated. Known excess amount of Quercetin was added to cap vial containing $2 \mathrm{ml}$ of different components of the SEDDS; oils, surfactants and co-surfactants. This was followed by vortexing the mixture for 2 minutes and then incubated in shaker water bath at $37{ }^{\circ} \mathrm{C}$ and $50 \mathrm{rpm}$ for 72 hours till equilibrium. Then the mixture was centrifuged at 3000 rpm for $5 \mathrm{~min}$ and excess insoluble Quercetin was discarded by filtration. The quantity of Quercetin in the supernatant from each component was determined using a validated HPLC method. The component of drug showing highest solubility was used for preparation of SEDDS.

\section{Construction of Ternary Phase Diagrams}

The results obtained from the solubility tests showed different SEDDS components (as oil, surfactants and co-surfactants) were chosen for construction of ternary phase diagram. In this study, different systems of the oil, surfactant, and cosurfactant were prepared at different ratios. The prepared systems were shaken to ensure complete mixing of the system components, and then specified volume of the system was poured into $250 \mathrm{ml}$ of 
distilled water under continuous stirring at a speed of 60 rpm. Systems were assessed visually for different characters as: the clarity of the formed aqueous dispersion, the time taken for emulsification, and the time needed for complete dispersion upon dilution. The obtained systems are visually evaluated following the grading system to identify the micro emulsifying regions [35];

- Grade A: Quickly formed emulsion of clear or bluish appearance.

- Grade B: Quickly formed but slightly less clear emulsion of bluish white appearance.

- Grade C: Fine and milky emulsion formed within 2 minutes.

- Grade D: Dull and greyish white emulsion of slightly oily appearance that is slow to emulsify takes more than 2 minutes.

- Grade E: Formulation, exhibiting either poor or slight emulsification with large oil globules on the surface.

\section{Preparation of Quercetin SEDDS}

After construction of the phase diagram and determination of the emulsified regions, SEDDS were prepared as per the component ratios. SEDDS components (oil, surfactant, and co-surfactant) in the specified ratios were weighed, accurately. Briefly, Quercetin was dissolved by co-surfactant, in glass vials. Oil and surfactants were accurately weighed into glass vials. The components were stirred gently and vortexing to mix than incubated at $37{ }^{\circ} \mathrm{C}$ until the drug dissolved perfectly. The prepared mixture was stored at room temperature.

\section{Characterization of QT-SEDDS}

\section{Droplet Size and Potential}

Optimized SEDDS was characterized for its droplet size and potential using Zeta Sizer after 2 and 6 $\mathrm{h}$ diluting $200 \mathrm{mg}$ oily mixture with $50 \mathrm{ml}$ deionized water, $\mathrm{pH}$ (1.2) and $\mathrm{pH}$ (6.8). In addition, the resultant emulsion after dilution was characterized for phase separation and drug precipitation.

\section{Determining Viscosity}

The rheological measurements of the prepared system were performed before and after dilution using Brookfield viscometer. Samples $(5 \mathrm{ml})$ were transferred to the instrument and allowed to equilibrate to $25 \pm{ }^{\circ} \mathrm{C}$ for 10 minutes prior to the measurement. The apparent viscosity was measured over a shear rate range of 0.1 $300 \mathrm{~s}^{-1}$. Viscosities ( $\mathrm{mPa} \mathrm{s}$ ) were determined for the prepared SEDDS at different shear rates. The mean of constant shear viscosity was calculated from the data obtained at $300 \mathrm{~s}^{-1}$.

\section{Freeze/thaw Stability}

Freeze/thaw stability of QT-SEDDS determined by exposing the formulation to three freeze/thaw cycles, which included freezing to $-20{ }^{\circ} \mathrm{C}$ for $24 \mathrm{~h}$ in a freezer followed by thawing at $40{ }^{\circ} \mathrm{C}$ for 24h. The formulations were then evaluated for the same parameters discussed above.

\section{In-vitro Dissolution Study}

In-vitro drug release from the prepared systems is an important criterion for the evaluation of prepared systems. The drug release from different SEDDS formulations studied using USP dissolution apparatus II. The prepared SEDDSs were filled into hard gelatin capsules. The release of Quercetin from the prepared SEDDSs and free Quercetin was carried out in simulated intestinal fluids (SGF, $\mathrm{pH} \mathrm{1.2,} \mathrm{and} \mathrm{SIF,} \mathrm{pH}$ 6.8). The apparatus was stirred at a rate of $100 \mathrm{rpm}$ and temperature was kept at $37^{\circ} \mathrm{C}$. At preset intervals, each sample of $5 \mathrm{ml}$ was withdraw and then replaced with an equal volume of fresh medium. Temperature was maintained each time to maintain the sink condition. The cumulative amount of drug released was analyzed for drug amount, spectrophotometrically.

\section{Accelerated Stability Studies}

The optimized formula of Quercetin was evaluated for accelerated stability for 9 months according to the ICH guidelines. The optimized formula was kept in screw-capped vials and stored under different storage conditions; in a stability chamber maintained at $25 \pm 2{ }^{\circ} \mathrm{C} / 55 \pm 5 \% \mathrm{RH}$ and $40 \pm 2{ }^{\circ} \mathrm{C} / 75 \pm$ $5 \%$ RH. Samples were then evaluated for the same parameters discussed above each 3 months.

\section{Phase II: Pharmacological study}

The optimized Quercetin-SEDDS formula was used for in-vivo studies. The in-vivo studies aim to look into the activity of the optimized formula as antidiabetic in streptozotocin-induced diabetic rats. The anti-diabetic activity of the prepared formula was compared to that of a standard official drug (glibenclamide). In this study, diabetes induced in adult male albino rats using streptozotocin. Male albino rats (200-250gm) were housed individually and light was maintained on a 12-h light/dark cycle. Animals retained in their house area for a week as an acclimatization period, with free access to food and water. After the acclimatization period, animals were randomly divided into 14 groups ( $\mathrm{n}=10$ in each group) as following:

- Group I: serves as normal (negative control), receive only distilled water $(0.2 \mathrm{ml} /$ rat $)$ orally for 30 successive days.

- Group II: serves as untreated diabetic (positive control), receive distilled water $(0.2 \mathrm{ml} / \mathrm{rat})$, orally for 30 successive days.

- Group III: treated with glibenclamide with a dose of $4.5 \mathrm{mg} / \mathrm{kg}$ orally for 30 successive days.

- Group IV: treated with Quercetin with a dose of $50 \mathrm{mg} / \mathrm{kg}$ orally for 30 successive days.

- Group V: treated with Quercetin with a dose of $100 \mathrm{mg} / \mathrm{kg}$ orally for 30 successive days.

- Group VI: treated with Quercetin with a dose of $200 \mathrm{mg} / \mathrm{kg}$ orally for 30 successive days. 
- Group VII: treated with optimized (SEDDS) formula of Quercetin with a dose of $50 \mathrm{mg} / \mathrm{kg}$ orally for 30 successive days.

- Group VIII: treated with optimized (SEDDS) formula of Quercetin with a dose of $100 \mathrm{mg}$ $/ \mathrm{kg}$ orally for 30 successive days.

- Group IX: treated with optimized (SEDDS) formula of Quercetin with a dose of $200 \mathrm{mg}$ $/ \mathrm{kg}$ orally for 30 successive days.

- Group X: treated with a combination of glibenclamide and Quercetin powder at the dose of $4.5 \mathrm{mg} / \mathrm{kg}$ and $100 \mathrm{mg} / \mathrm{kg}$ respectively orally for 30 successive days.

- Group XI: treated with a combination of glibenclamide and optimized (SEDDS) formula of Quercetin with a dose of $4.5 \mathrm{mg} / \mathrm{kg}$ and $100 \mathrm{mg} / \mathrm{kg}$, respectively, orally for 30 successive days.

- Group XII: treated with onion (fresh red onion) with a dose of $50 \mathrm{mg} / \mathrm{kg}$ orally for 30 successive days.

- Group XIII: treated with onion (fresh red onion) with a dose of $100 \mathrm{mg} / \mathrm{kg}$ orally for 30 successive days.

- Group XIV: treated with onion (fresh red onion) with a dose of $200 \mathrm{mg} / \mathrm{kg}$ orally for 30 successive days

\section{Experimental induction of diabetes in rats}

Animals were retained in fasting condition for at least 18 hours through night. Each rat was injected by $0.5 \mathrm{ml}$ of dextrose $5 \%$ just $15 \mathrm{~min}$ prior Streptozotocin injection. Streptozotocin (STZ) as a single intraperitoneal dose in a ratio of $55 \mathrm{mg} / \mathrm{kg}$ of body weight was dissolved in $0.1 \mathrm{M}$ freshly prepared cold citrate buffer ( $\mathrm{pH} 4.5)$ and injected into each rat of all groups except group I. The control animals (group I) received an equivalent amount of citrate buffer only. A stable hyperglycemia was developed in rats by fasting of 16 hours, confirmed by the blood glucose measurement in the blood of tail vein, checked in 48 hours after STZ administration, using portable glucometer (Accu-Chek, Roche, Germany). Rats with fasting blood glucose of greater than $250 \mathrm{mg} / \mathrm{dl}$ were considered diabetic and used in further study.

\section{Collection of blood samples and Biochemical analysis}

At the end of this experiment, the rats were kept in fasting condition for 18 hours through night and blood was drawn from retro-orbital plexus of the rats. The blood was transferred to Eppendorf tubes containing heparin sodium $(20 \mathrm{U} / \mathrm{mL}$ blood $)$ prepared in $0.9 \%$ saline, left for 20 minutes at room temperature, centrifuged at $3000 \mathrm{rpm}$ for 20 minutes to separate the serum for measuring the following parameters:

- Blood glucose level after fasting measured using a portable glucometer (Accu-Chek, Roche, Germany).

- $\quad$ Lipid profile (total cholesterol, LDL, HDL and triglycerides) was estimated spectrophotometrically using standard enzymatic kits.

- Lipid peroxidation was measured as malondialdehyde (MDA).

- Serum reduced glutathione (GSH), catalase (CAT) and superoxide dismutase (SOD) were determined following the standard protocols.

Furthermore, the animals were anesthetized, sacrificed, and the abdomen was opened. The organs excised for histological examination include pancreas, liver, and kidney. The organ tissues were isolated and fixed in 10\% neutral buffered formalin and dehydrated through a graded alcohol series, embedded in paraffin blocks and sections of $5 \mu \mathrm{m}$ were prepared. The sections were stained in hematoxylin and eosin that were evaluated for histological changes in these organs.

\section{STATISTICAL ANALYSIS}

All the results were expressed as the mean \pm standard deviation (SD). Statistical analysis was performed using Sigma Stat (version 2.03) applying one-way ANOVA followed by Turkey-Kramer multiple comparison test. A value of $\mathrm{P}<0.05$ showed statistically significant difference.

\section{RESULTS AND DISCUSSION}

The results of biochemical parameters notably deteriorated among the diabetic rats as compared to the normal control group after 30 days (Table-1). Quercetin $(10$ and $50 \mathrm{mg} / \mathrm{kg}$ ) and fresh red onion treatments enhanced the biochemical parameters, evidently. There were no evident differences in the levels of HDL-C between the study groups.

Oral administration of flavonoids decreased significantly in plasma glucose levels of normoglycemic rats, as compared to the control group. Bes activity was shown by Quercetin and fresh red onion after 2 and 4 weeks, respectively. Quercetin showed sugar lowering activity profile, in comparison to glibenclamide (Fig-1).

Figure-2 shows that glibenclamide, Quercetin, optimized (SEDDS) formula of Quercetin, glibenclamide combined with Quercetin powder, glibenclamide combined with optimized (SEDDS) formula of Quercetin, and fresh red onion produced significant reduction in the increased level of blood glucose after administration of glucose.

Figure-3 shows acute oral administration of four flavonoids (at 50, 100 and $200 \mathrm{mg} / \mathrm{kg}$ ) reduce plasma glucose levels significantly as compared to control group. Therefore, Quercetin was considered among the active compound, as compared to antidiabetic effect showed by glibenclamide. 
There are many traditional plants used in the treatment of diabetes mellitus. Medical system is greatly challenged for managing diabetes without any side effects. This has increasing demand of natural products of less side effects and anti-diabetic activity. There are many plant and herbs products that exhibit hypoglycemic action, and flavonoid is among them. It has emerged as a bioactive anti-diabetic. The present in vivo study was conducted among normal rats for hypoglycemic effect of flavonoid compounds. The results of the research shows a significant reduction in blood glucose levels for quercetin, which was maximum at $4^{\text {th }}$ week. This might be due to inhibiting activity of glucose transporter and increase peripheral utilization of glucose from intestine. The study clarifies that compounds selected in this study increase glucose uptake by enhancing peripheral glucose utilization either by the mediation of enhanced insulin secretion, direct stimulation of glucose uptake or inhibiting the glucose transporter activity.

Table-1: Biochemical parameters of experimental diabetic rats after 30 days of different natural flavonoids

\begin{tabular}{|l|l|l|l|l|l|}
\hline Group & Fasting blood glucose & Total cholesterol & LDL & HDL & Triglycerides \\
\hline I & $5.27 \pm 0.31$ & $1.41 \pm 0.25$ & $0.31 \pm 0.08$ & $0.62 \pm 0.12$ & $0.69 \pm 0.13$ \\
\hline II & $15.71 \pm 0.71$ & $1.36 \pm 0.18$ & $0.535 \pm 0.16$ & $0.58 \pm 0.07$ & $1.13 \pm 0.53$ \\
\hline III & $25.26 \pm 3.45$ & $4.33 \pm 1.2$ & $0.98 \pm 0.37$ & $0.70 \pm 0.19$ & $4.61 \pm 1.33$ \\
\hline IV & $21.76 \pm 2.61$ & $1.39 \pm 0.31$ & $0.74 \pm 0.04$ & $0.56 \pm 0.12$ & $1.08 \pm 0.59$ \\
\hline V & $22.98 \pm 2.16$ & $1.49 \pm 0.41$ & $0.635 \pm 0.26$ & $0.68 \pm 0.17$ & $0.98 \pm 0.49$ \\
\hline VI & $21.98 \pm 1.16$ & $1.12 \pm 0.19$ & $0.88 \pm 0.27$ & $0.60 \pm 0.09$ & $1.06 \pm 0.66$ \\
\hline VII & $16.71 \pm 0.81$ & $4.33 \pm 1.29$ & $0.635 \pm 0.26$ & $0.59 \pm 0.20$ & $1.03 \pm 0.63$ \\
\hline VIII & $24.26 \pm 2.45$ & $3.33 \pm 1.19$ & $4.35 \pm 1.58$ & $0.49 \pm 0.10$ & $0.97 \pm 0.50$ \\
\hline IX & $25.26 \pm 3.45$ & $1.49 \pm 0.41$ & $5.35 \pm 1.68$ & $0.66 \pm 0.22$ & $0.96 \pm 0.56$ \\
\hline X & $4.27 \pm 0.21$ & $1.31 \pm 0.15$ & $0.21 \pm 0.07$ & $0.72 \pm 0.22$ & $2.03 \pm 1.64$ \\
\hline XI & $22.76 \pm 3.61$ & $2.46 \pm 0.38$ & $0.84 \pm 0.14$ & $0.78 \pm 0.28$ & $0.59 \pm 0.12$ \\
\hline XII & $17.71 \pm 0.91$ & $2.49 \pm 0.51$ & $0.735 \pm 0.36$ & $0.72 \pm 0.23$ & $5.61 \pm 3.23$ \\
\hline XIII & $23.76 \pm 4.61$ & $3.12 \pm 0.39$ & $0.94 \pm 0.24$ & $0.77 \pm 0.22$ & $3.61 \pm 1.23$ \\
\hline XIV & $23.98 \pm 3.16$ & $5.33 \pm 2.29$ & $1.08 \pm 0.47$ & $0.78 \pm 0.37$ & $0.70 \pm 0.33$ \\
\hline
\end{tabular}

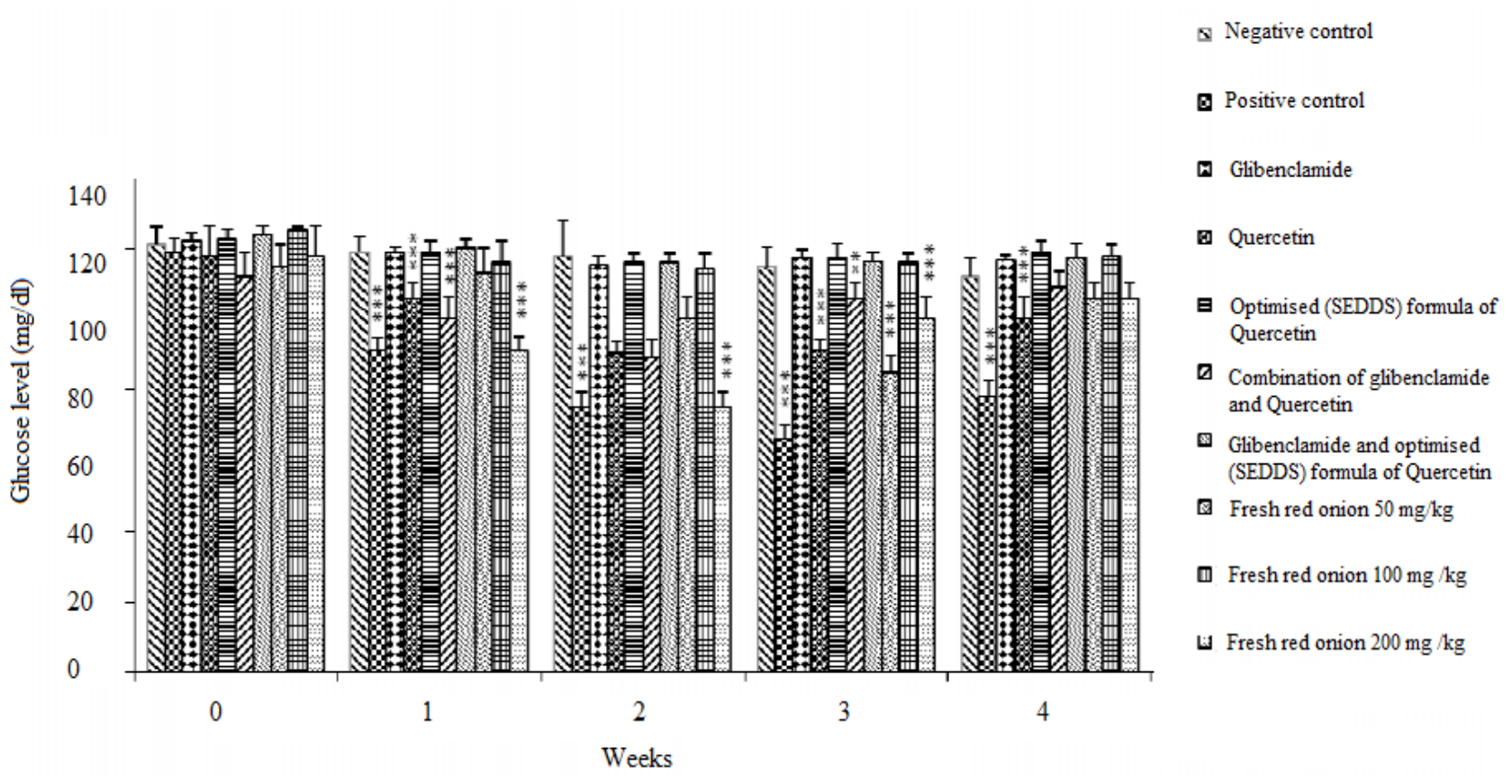

Fig-1: Acute in vivo hypoglycemic activity of flavonoids 


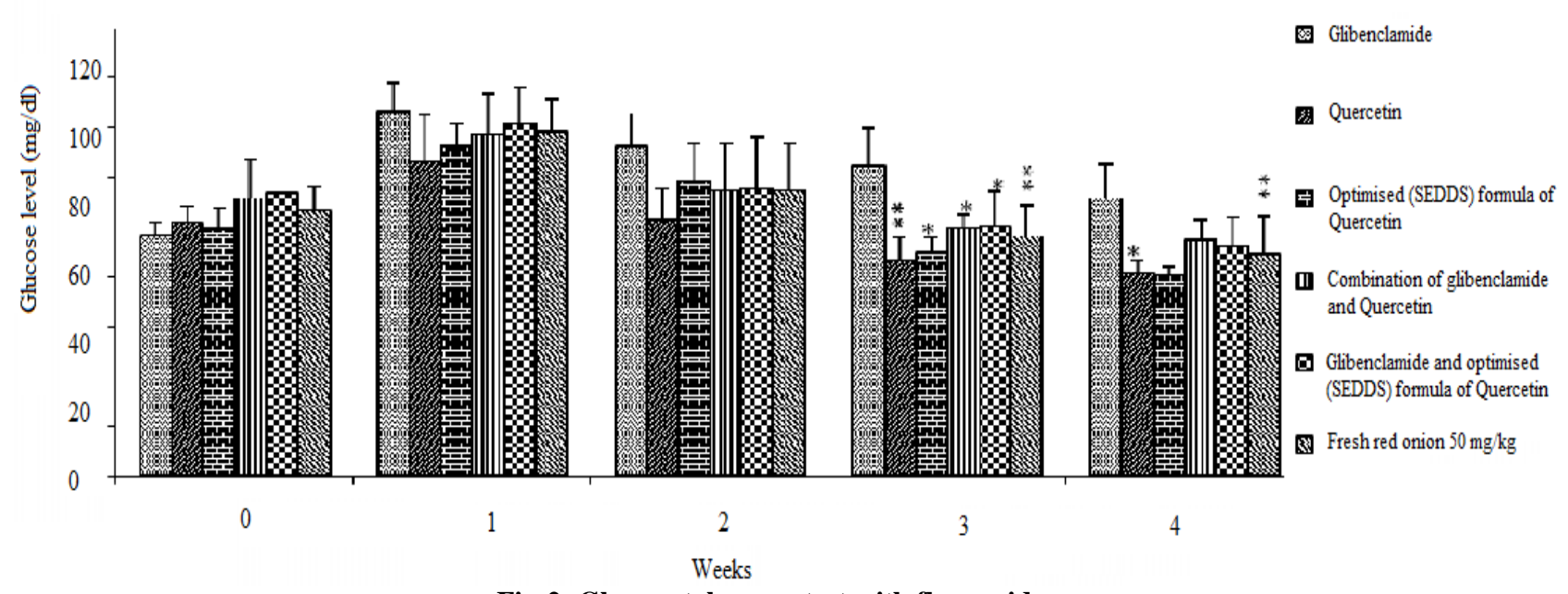

Fig-2: Glucose tolerance test with flavonoids

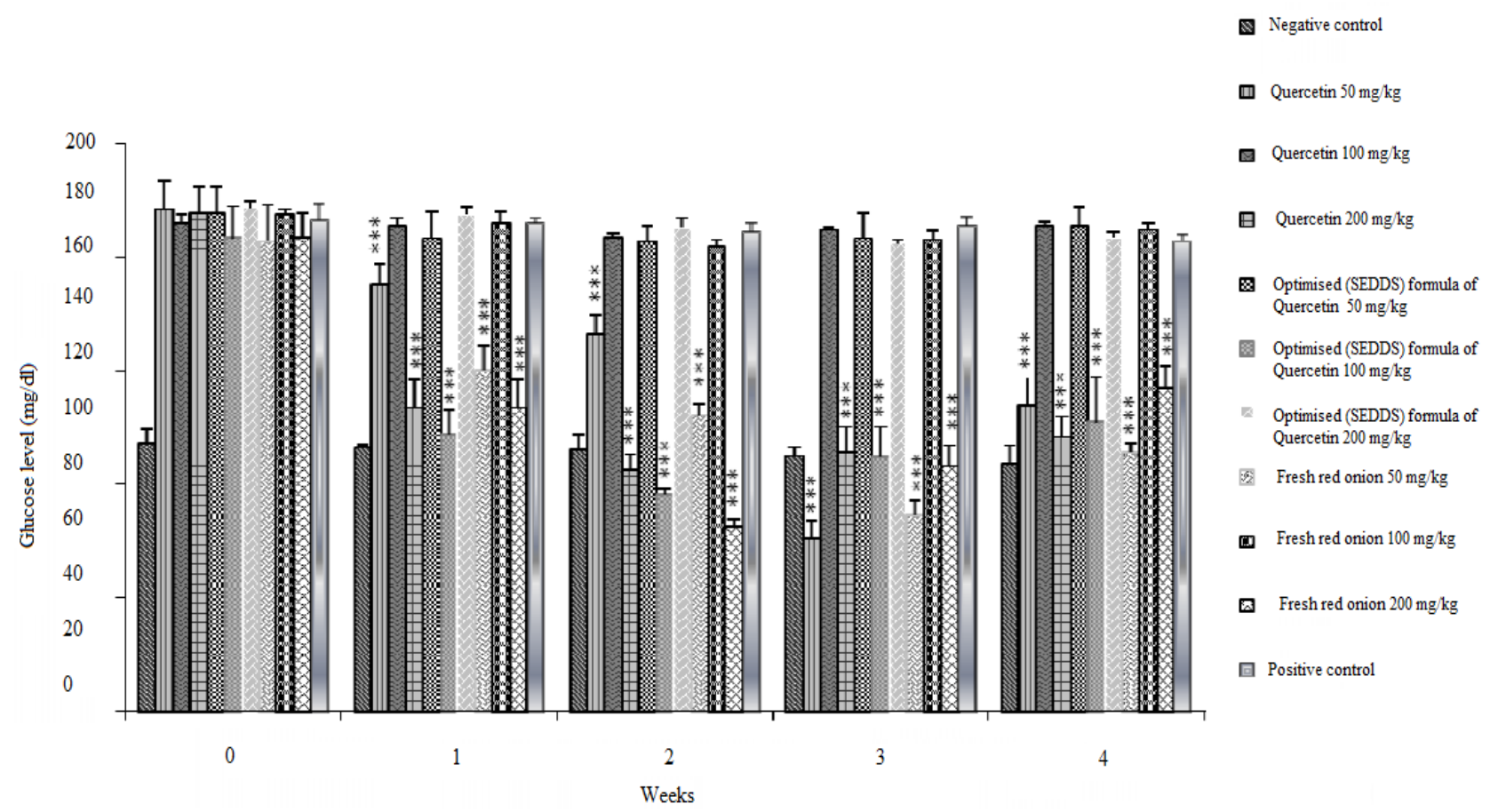

Fig-3: Anti-diabetic activity with $50 \mathrm{mg} / \mathrm{kg}$ and $100 \mathrm{mg} / \mathrm{kg}$ dose of flavonoids

\section{CONCLUSION}

The present study concluded that flavonoids are important in lowering the blood glucose as it stimulates $\beta$-cells to release more insulin, however, in this we have only conducted comparative hypoglycemic and anti-diabetic activity among glibenclamide, Quercetin, and fresh red onion. The study can be extended in future considering other mechanisms that relate with the anti-diabetic actions and have not been covered in this study.

\section{REFERENCES}

1. Ahmed, M. F., Kazim, S. M., Ghori, S. S., Mehjabeen, S. S., Ahmed, S. R., Ali, S. M., \& Ibrahim, M. (2010). Antidiabetic activity of Vinca rosea extracts in alloxan-induced diabetic rats. International

Journal

of

2. Kumar, V., Ahmed, D., Gupta, P. S., Anwar, F., \& Mujeeb, M. (2013). Anti-diabetic, anti-oxidant and anti-hyperlipidemic activities of Melastoma malabathricum Linn. leaves in streptozotocin induced diabetic rats. BMC complementary and alternative medicine, 13(1), 1-19.

3. Kumar, G. P. S., Arulselvan, P., Kumar, D. S., \& Subramanian, S. P. (2006). Anti-diabetic activity of fruits of Terminalia chebula on streptozotocin induced diabetic rats. Journal of health science, 52(3), 283-291.

4. Sumana, G., \& Suryawashi, S. A. (2001). Effect of vinca rosa extract in treatment of alloxan diabetes 
in male albino rats. Indian Journal of Experimental Biology. 39:748-759.

5. Holman, R. R., \& Turner, R. C. (1991). Oral agents and insulin in the treatment of NIDDM. Textbook of Diabetes. Oxford: Blackwell, 9, 467-469, Blackwell, Oxford, UK.

6. Rao, B. K., Kesavulu, M. M., \& Apparao, C. H. (2001). Antihyperglycemic activity of Momordica cymbalaria in alloxan diabetic rats. Journal of ethnopharmacology, 78(1), 67-71.

7. Kameswrarao, B., Giri, R., Kesavulu, M. M., \& Apparao, C. H. (1997). Herbal medicine. In The Management by Indigenous Resources, Bajaj J. S., Ed., 375-377, Diabetes Mellitus in Developing Countries. Interprint, New Delhi, India.

8. Baynes, J. W. (1991). Role of oxidative stress in development of complications in diabetes. Diabetes, 40(4), 405-412.

9. Baynes, J. W. (1995). Reactive oxygen in the etiology and complications of diabetes. In Drug, Diet and Disease: Mechanistic Approach to Diabetes, vol. 2. Edited by Loannides, C., Flatt, P. R., Hertfordshire: Ellis Horwood Limited; 203231.

10. Nabi, S. A., Kasetti, R. B., Sirasanagandla, S., Tilak, T. K., Kumar, M. V. J., \& Rao, C. A. (2013). Antidiabetic and antihyperlipidemic activity of Piper longum root aqueous extract in STZ induced diabetic rats. BMC complementary and alternative medicine, 13(1), 1-9.

11. Midhet, F. M., Al-Mohaimeed, A. A., \& Sharaf, F. K. (2010). Lifestyle related risk factors of type 2 diabetes mellitus in Saudi Arabia. Saudi Med $J, 31(7), 768-774$.

12. Moller, D. E. (2001). New drug targets for type 2 diabetes and the metabolic syndrome. Nature, 414(6865), 821-827.

13. Alqurashi, K. A., Aljabri, K. S., \& Bokhari, S. A. (2011). Prevalence of diabetes mellitus in a Saudi community. Annals of Saudi medicine, 31(1), 1923.

14. Lewis, D., Udupa, N., \& Atin, K. (2009). Solid dispersions: a review. Pak J Pharm Sci,22(2), 234-46.

15. Gursoy, R. N., \& Benita, S. (2004). Selfemulsifying drug delivery systems (SEDDS) for improved oral delivery of lipophilic drugs. Biomedicine \& pharmacotherapy, 58(3), 173-182.

16. Tamilvanan, S., \& Benita, S. (2004). The potential of lipid emulsion for ocular delivery of lipophilic drugs. European Journal of Pharmaceutics and Biopharmaceutics, 58(2), 357-368.

17. Gursoy, N., Garrigue, J. S., Razafindratsita, A., Lambert, G., Benita, S., \& Bloom, D. R. (2003). Excipient effects on in vitro cytotoxicity of a novel paclitaxel self- emulsifying drug delivery system. Journal of Pharmaceutical Sciences, 92(12), 2411-2418.
18. Ichikawa, H., Watanabe, T., Tokumitsu, H., \& Fukumori, Y. (2007). Formulation considerations of gadolinium lipid nanoemulsion for intravenous delivery to tumors in neutron-capture therapy. Current drug delivery, 4(2), 131-140.

19. Patel, H., Suhagia, B., Shah, S., Rathod, I., \& Parmar, V. (2007). Preparation and characterization of etoricoxib- $\beta$-cyclodextrin complexes prepared by the kneading method. Acta pharmaceutica, 57(3), 351-359.

20. El-laithy, H. M., Hamza, Y. E., \& Kandil, S. M. (2011). Design and hepatoprotective evaluation of biphenyl dimethyl dicarboxylate (DDB) and silymarin solid dispersion and self-micro emulsifying drug delivery systems. Life Science Journal, 8(1), 298-310.

21. Chouksey, R. A. J. E. N. D. R. A., Pandey, H., Jain, A. K., Soni, H. I. M. E. S. H., \& Saraogi, G. K. (2011). Preparation and evaluation of the selfemulsifying drug delivery system containing atorvastatin HMG-CoA inhibiter. Int $J$ Pharm Pharm Sci, 3(3), 147-152.

22. Tang, J., Sun, J., Cui, F., \& He, Z. (2006). Preparation of self-emulsifying drug delivery systems of Ginkgo biloba extracts and in vitro dissolution studies. 亚洲传统医药, 1(3-4), 138141.

23. Wadhwa, J., Nair, A., \& Kumria, R. (2012). Emulsion forming drug delivery system for lipophilic drugs. Acta Pol Pharm, 69(2), 179-91.

24. Li, P., Ghosh, A., Wagner, R. F., Krill, S., Joshi, Y. M., \& Serajuddin, A. T. (2005). Effect of combined use of nonionic surfactant on formation of oil-in-water microemulsions. International journal of pharmaceutics, 288(1), 27-34.

25. Derakhshanian, H., Djalali, M., Djazayery, A., Golpaie, A., Ekhlasi, G., \& Hosseinzadeh-Attar, M. J. (2012). Quercetin Improves Postprandial Hyperglycemia in Rats Treated With High-Dose Glucocorticoid. Pharmacology Online, 2, 93-97.

26. Kim, J. H., Kang, M. J., Choi, H. N., Jeong, S. M., Lee, Y. M., \& Kim, J. I. (2011). Quercetin attenuates fasting and postprandial hyperglycemia in animal models of diabetes mellitus. Nutrition research and practice, 5(2), 107-111.

27. Kim, S. H., Jo, S. H., Kwon, Y. I., \& Hwang, J. K. (2011). Effects of onion (Allium cepa L.) extract administration on intestinal $\alpha$-glucosidases activities and spikes in postprandial blood glucose levels in SD rats model. International journal of molecular sciences, 12(6), 3757-3769.

28. Khonkarn, R., Mankhetkorn, S., Hennink, W. E., \& Okonogi, S. (2011). PEG-OCL micelles for quercetin solubilization and inhibition of cancer cell growth. European journal of pharmaceutics and biopharmaceutics, 79(2), 268-275.

29. Zheng, Y., Haworth, I. S., Zuo, Z., Chow, M. S., \& Chow, A. H. (2005). Physicochemical and structural characterization of quercetin- $\beta$ - 
cyclodextrin complexes. Journal of pharmaceutical sciences, 94(5), 1079-1089.

30. Sri, K. V., Kondaiah, A., Ratna, J. V., \& Annapurna, A. (2007). Preparation and characterization of quercetin and rutin cyclodextrin inclusion complexes. Drug development and industrial pharmacy, 33(3), 245253.

31. Khaled, K. A., \& Mahrous, G. M. (2001). Comparative Study of the Dissolution and Physicochemical Characteristics of the Binary Systems of Quercetin with Polyethylene Glycol, Polyvinylpyrrolidone, and Hydroxyproply-betacyclodextrin. Saudi Pharmaceutical Journal, 9(1), 34-42.

32. Sahoo, N. G., Kakran, M., Shaal, L. A., Li, L., Müller, R. H., Pal, M. L. P. T., \& Tan, L. P.
(2011). Preparation and characterization of quercetin nanocrystals. Journal of pharmaceutical sciences, 100(6), 2379-2390.

33. Kakran, M., Sahoo, N. G., Li, L., \& Judeh, Z. (2012). Fabrication of quercetin nanoparticles by anti-solvent precipitation method for enhanced dissolution. Powder Technology, 223, 59-64.

34. Zheng, Y., \& Chow, A. H. (2009). Production and characterization of a spray-dried hydroxypropyl- $\beta$ cyclodextrin/quercetin complex. Drug development and industrial pharmacy, 35(6), 727734.

35. Gupta, A. K., Mishra, D. K., \& Mahajan, S. C. (2011). Preparation and in-vitro evaluation of self emulsifying drug delivery system of antihypertensive drug valsartan. Int. J. Pharm. Life Sci, 2(3), 633-639. 